\title{
Characterization of in band stray light in SBUV/2 instruments
}

\author{
L. K. Huang ${ }^{1}$, M. T. DeLand ${ }^{1}$, S. L. Taylor ${ }^{1}$, and L. E. Flynn ${ }^{2}$ \\ ${ }^{1}$ Science Systems and Applications, Inc. (SSAI), 10210 Greenbelt Road, Suite 600, Lanham, Maryland 20706, USA \\ ${ }^{2}$ NOAA NESDIS, College Park, Maryland, USA \\ Correspondence to: L. K. Huang (liang-kang.huang@ssaihq.com)
}

Received: 22 July 2013 - Published in Atmos. Meas. Tech. Discuss.: 28 August 2013

Revised: 22 November 2013 - Accepted: 17 December 2013 - Published: 28 January 2014

\begin{abstract}
Significant in-band stray light (IBSL) error at solar zenith angle (SZA) values larger than $77^{\circ}$ near sunset in 4 SBUV/2 (Solar Backscattered Ultraviolet) instruments, on board the NOAA-14, 17, 18 and 19 satellites, has been characterized. The IBSL error is caused by large surface reflection and scattering of the air-gapped depolarizer in front of the instrument's monochromator aperture. The source of the IBSL error is direct solar illumination of instrument components near the aperture rather than from earth shine. The IBSL contamination at $273 \mathrm{~nm}$ can reach $40 \%$ of earth radiance near sunset, which results in as much as a $50 \%$ error in the retrieved ozone from the upper stratosphere. We have analyzed SBUV/2 albedo measurements on both the dayside and nightside to develop an empirical model for the IBSL error. This error has been corrected in the V8.6 SBUV/2 ozone retrieval.
\end{abstract}

\section{Introduction}

Stratospheric ozone shields Earth's biosphere from solar ultraviolet radiation (DeFabo, 2000), while tropospheric ozone as a pollutant impacts global health and economy (Selin et al., 2009). Atmospheric ozone plays an important role in climate change (Fiore et al., 2002). The Solar Backscattered U1traviolet (SBUV) program for monitoring atmospheric ozone profiles has spanned more than three decades (Stolarski and Frith, 2006). The first SBUV instrument was launched on the Nimbus-7 satellite into a sun synchronized polar orbit in 1978 (Heath et al., 1975). It has been followed by 7 SBUV/2 instruments on NOAA satellites from 1985 to the present day (NOAA-9, NOAA-11, NOAA-14, NOAA16, NOAA-17, NOAA-18 and NOAA-19) (Frederick et al., 1986; McPeters et al., 2013). The last 4 SBUV/2 instruments are still active. SBUV/2 instruments measure backscattered terrestrial radiance in the nadir direction at $12 \mathrm{UV}$ wavelengths from 252 to $340 \mathrm{~nm}$ with a nominal spectral bandpass of $1.13 \mathrm{~nm}$ FWHM (full width at half maximum) for ozone monitoring. The incident solar irradiance at the top of atmosphere is also measured by SBUV/2 instruments on a regular basis for calibration. Measured ratios of the earth radiance to the solar irradiance (albedo) are used to derive ozone profiles (Bhartia et al., 1996, 2013). The long-term SBUV ozone record provides important information to scientific research in climatology and meteorology, and to environmental protection efforts (Stolarski and Frith, 2006; Terao and Logan, 2007).

In the SBUV/2 spectral range, the earth radiance intensity varies by more than 3 orders of magnitude. Figure 1 shows typical spectra of radiance and albedo measured by an SBUV/2 instrument in its continuous spectral scan mode, which were taken and averaged in latitude bands from $15^{\circ} \mathrm{S}$ to $15^{\circ} \mathrm{N}$. The square signs indicate the 12 discrete SBUV/2 wavelengths for the regular ozone monitoring operation. Light at the short wavelength channels (252-298 nm), mostly absorbed by ozone before reaching ground, provides information of ozone density in the stratosphere (Bhartia et al., 2013). The measurements at longer wavelengths, which can reach the surface, provide limited information about the ozone distribution at low altitude. Detecting a $2 \%$ ozone density change in Umkehr layers in the stratosphere requires the calibration of SBUV/2 albedo measurements at the short wavelengths to be accurate to within $1 \%$. With such a large dynamical signal range and stringent accuracy requirement, stray light often becomes a difficult issue in optical design and characterization of a spectrometer. There are 2 types of stray light, out of band stray light (OBSL) and in-band stray light (IBSL). OBSL represents contamination of the signal 


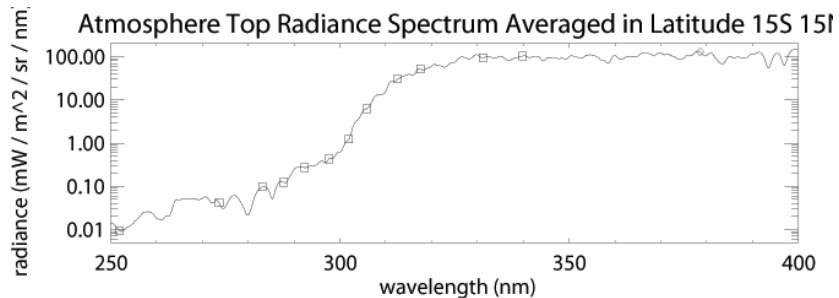

Atmosphere Top Albedo Spectrum Averaged in Latitude 15S 15N

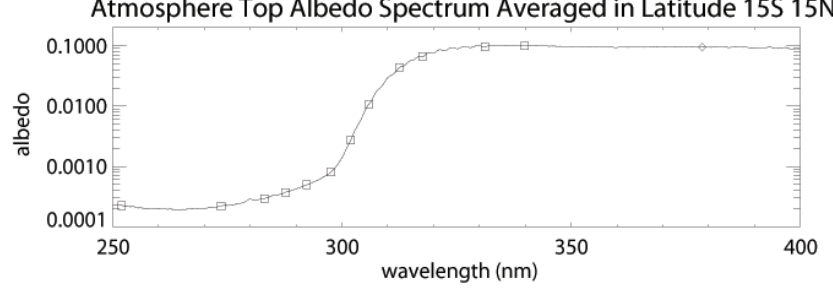

Fig. 1. Solar backscattered ultraviolet spectrum measured by NOAA-16 SBUV/2. The monochromator completes a continuous spectral scan from 405 to $160 \mathrm{~nm}$ in $168 \mathrm{~s}$. The radiance spectrum (top panel) is an average of 60 scans between the latitudes of $15^{\circ} \mathrm{S}$ and $15^{\circ} \mathrm{N}$, which were taken during four consecutive orbits once per week in November 2001. The albedo spectrum (bottom panel), equal to the radiance spectrum divided by the solar irradiance spectrum, is used in the ozone retrieval. Square symbols represent SBUV/2 discrete wavelengths used for ozone measurements in a $32 \mathrm{~s}$ scan cycle. The diamond shows the location of the cloud cover radiometer (CCR) measurements.

at the measured wavelength from light at other wavelengths due to imperfect spectral filtering. In the SBUV ozone measurements, a typical OBSL error for bright surface scenes can add a few percent error in radiance at the shortest wavelength (DeLand et al., 2012). A simplified description of the OBSL characterization is presented in that paper, but a detailed discussion would require substantially more space. We will not discuss the characterization of OBSL in this paper. IBSL is considered to represent imperfect spatial filtering of external illumination that passes through the spectral bandpass of a given wavelength channel. Analysis of stray light in a remote sensing instrument is often an important part of sensor characterization. When an IBSL error is present, the sensor's measurements are contaminated with light that does not come from the research target. The signal is an additive error that cannot be canceled in the albedo ratio in the ozone retrieval. As will be described later in this paper, the IBSL error is also wavelength dependent as well as time dependent. Typical retrieval algorithms for atmosphere trace gasses could not reduce or eliminate such additive errors in their data products. Therefore, detection, characterization and correction of IBSL, if significant, are necessary. We hope that our study of SBUV IBSL can also provide lessons for the design of future instruments.

SBUV/2 instruments have an $11^{\circ}$ (FWHM) field of view, which projects to approximately a $168 \mathrm{~km} \times 168 \mathrm{~km}$ square on the ground in the nadir direction. The response over this
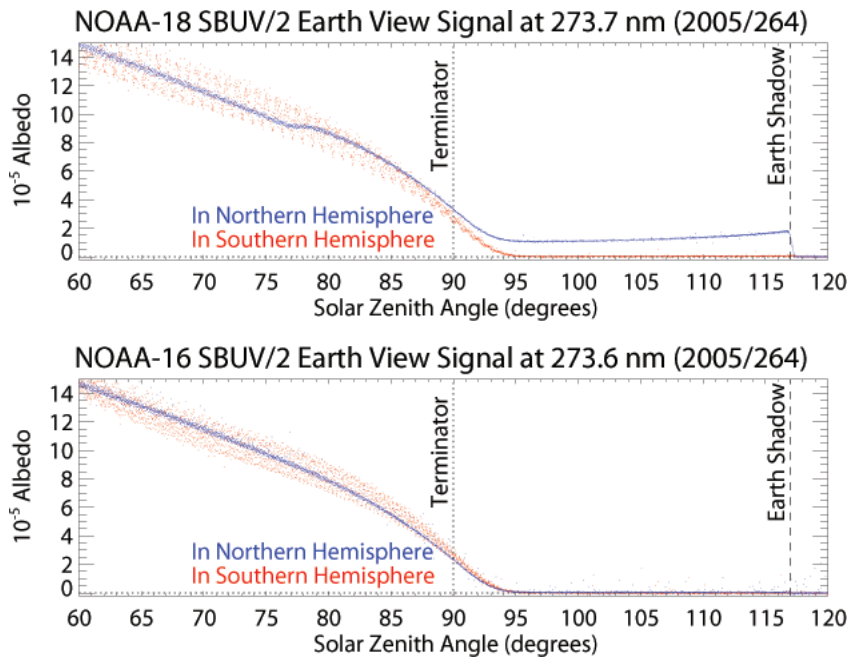

Fig. 2. Comparison of SBUV/2 earth view (radiance) signals at $273 \mathrm{~nm}$ between NOAA-18 (top panel) and NOAA-16 (bottom panel). The IBSL error in NOAA-16 data is negligible. NOAA-18 SBUV/2 data have significant IBSL errors at SZA $>77^{\circ}$ approaching the terminator near North Pole. NOAA-16 and NOAA-18 orbits were in proximity at the Equator crossing local times of 14:59 and $14: 01$, respectively, to minimize diurnal difference in the earth radiance.

field of view was characterized in the SBUV/2 prelaunch calibrations. The instrument's sensitivity varies less than $10 \%$ within a $10^{\circ} \times 10^{\circ}$ center area, then, decreases sharply by a factor of $10^{-4}$ within $2^{\circ}$ outside the center area. Such performance makes the SBUV radiance measurements well defined in geolocation. Prelaunch testing did not fully simulate the solar light direct illumination of surfaces on the spacecraft near the SBUV/2 instrument's aperture, which happens at an incidence angle nearly perpendicular to the instrument's optical axis. The solar flux is 4 orders of magnitude more intense than the earth radiance at $252 \mathrm{~nm}$, as shown by the albedo values in the bottom panel in Fig. 1. Figure 2 shows sample albedo measurements at $273 \mathrm{~nm}$ on the same date by NOAA18 SBUV/2 (N18) in the top panel and NOAA-16 SBUV/2 (N16) in the bottom panel. We will use the abbreviation N9, N11, N14, N16, N17, N18 and N19 for SBUV/2 instruments on board the NOAA-9, 11, 14, 16, 17, 18 and 19 satellites, respectively. Both N16 and N18 were emerging from darkness into daylight in the Southern Hemisphere and moving into darkness in the Northern Hemisphere in the same week around the fall equinox in 2005, in orbits with Equator crossing local times of 14:59 and 14:01, respectively. We selected two instruments with similar orbits to minimize radiance differences due to diurnal ozone variations. The N16 signal monotonically decreases in both hemispheres as solar zenith angle (SZA) increases and approaches zero at $\mathrm{SZA}=95^{\circ}$ and beyond. This is consistent with expectations for a short wavelength $(\lambda<300 \mathrm{~nm})$ that is blocked by ozone absorption before reaching ground. The N18 Southern Hemisphere 


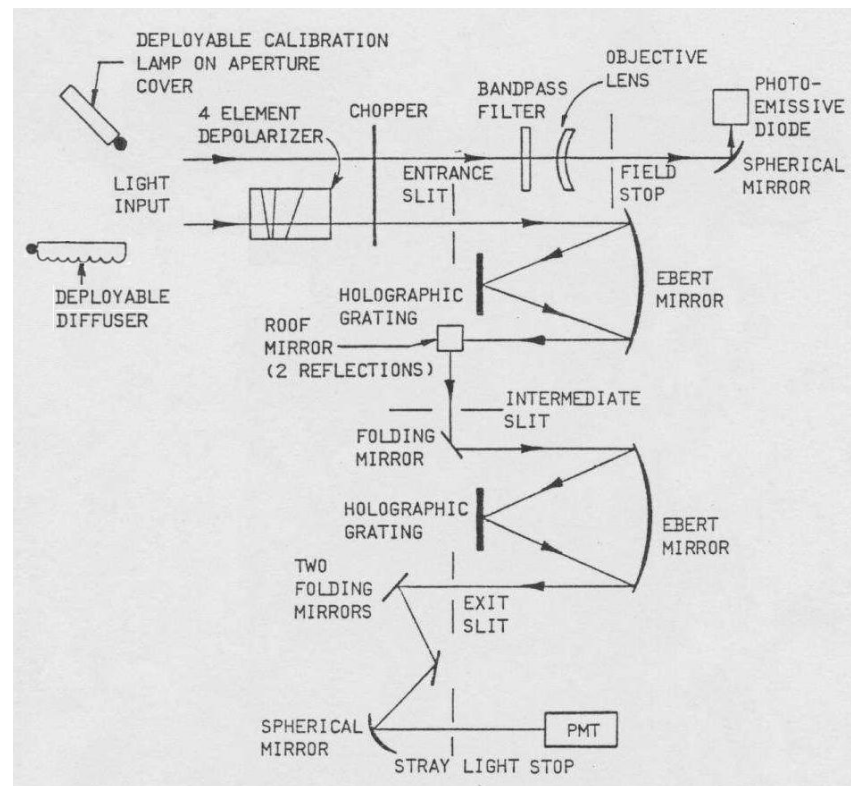

Fig. 3. Diagram of SBUV/2 instrument optics. SBUV(/2) instruments consist of double Ebert monochromators for scanning ozone absorption wavelengths, and a bandpass-filter photometer as a cloud cover monitor. A depolarizer is installed in front of the monochromator to minimize the polarization effect with atmospheric Rayleigh scattering coupled with strong polarity with the grating spectrometer. Courtesy of Ball Aerospace and Technologies Corp.

data are consistent with N16, but the N18 Northern Hemisphere data show a sharp step increase between $\mathrm{SZA}=78^{\circ}$ and $80^{\circ}$ in comparison with N16. The increased signal is still present in nightside data until the satellite passes into Earth's shadow (eclipse) at $\mathrm{SZA}=118^{\circ}$. This behavior suggests that the source of the anomalous signal is direct solar illumination of the SBUV/2 instrument. We found significant IBSL contamination in the earth radiance measurements at the short wavelengths with N14, N17, N18 and N19 at SZAs greater than $78^{\circ}$ approaching the terminator. The magnitude of the IBSL contamination in the radiance measurements is as high as $25-50 \%$ at $\mathrm{SZA}=88^{\circ}$. In order to accurately retrieve stratospheric ozone in these situations, which frequently occur in polar regions, it is necessary to correct the IBSL error in the SBUV/2 radiance measurements. This paper reports our analysis and characterization of the SBUV/2 IBSL error in orbit using N19 as an example, which has the largest IBSL error among SBUV/2 instruments for an easy illustration.

\section{$2 \mathrm{SBUV} / 2$ instrument}

Figure 3 is a schematic diagram of the SBUV/2 optical design. SBUV/2 instruments are equipped with double EbertFastie monochromators, which can block OBSL at an extinction rate of $10^{-6}$. The monochromator covers a wavelength range from 160 to $405 \mathrm{~nm}$, with a nominal spectral bandpass of $1.13 \mathrm{~nm}$ FWHM. SBUV/2 instruments make discrete measurements for profile ozone monitoring at nominal wavelengths of 252.0, 273.7, 283.2, 287.7, 292.4, 297.6, 302.0, $305.9,312.7,317.6,331.3$ and $339.9 \mathrm{~nm}$ (except for Nimbus$7 \mathrm{SBUV}$, which had channel 1 at $255.7 \mathrm{~nm}$ ). It takes $24 \mathrm{~s}$ to scan through these 12 wavelengths, and completes the scan cycle in $32 \mathrm{~s}$. The cloud cover radiometer (CCR), installed side-by-side with the monochromator, makes continuous and simultaneous measurements at $378.6 \mathrm{~nm}$ with a $3 \mathrm{~nm}$ band pass to monitor earth surface reflectivity change during the spectral scan. A depolarizer in front of the monochromator is used to eliminate the polarization effects of atmospheric Rayleigh scattering, concerning strong polarity of a grating spectrometer that acts as an analyzer. A diffuser plate can be deployed to reflect the solar light into the sensor's aperture after terminator crossing to measure the solar irradiance. This measurement is performed every day during normal operations. Since the radiance measurement and the irradiance measurement share the same monochromator and CCR, the sensor's sensitivity is canceled in the albedo ratio used for ozone retrieval. Therefore, the bidirectional reflectance distribution function (BRDF) calibration of the solar diffuser, the only different element in the optical paths between the radiance and irradiance measurements, is more important than the absolute photometric calibration for the ozone retrieval. Rigorous SBUV/2 albedo calibrations are performed in laboratory before launch, and are traceable to NIST (National Institute of Standards and Technology) BRDF standards. The SBUV/2 diffuser reflectivity changes, from prelaunch tests to on-orbit conditions, and over years of operation, are monitored using emission lines from an on-board mercury lamp. Typically, the diffuser reflectivity at $250 \mathrm{~nm}$ degraded at a relative annual rate of no more than $3 \%$ per year, with significantly smaller changes observed in the long wavelengths. The diffuser calibration measurements in orbit were scheduled on a weekly basis to ensure the accuracy of the albedo calibration. Details of SBUV/2 calibrations can be found elsewhere in literature (Frederick et al., 1986; Heath et al., 1993; Hilsenrath et al., 1995; Janz et al., 1995; DeLand et al., 2012).

Figure 4 shows a picture of an SBUV/2 instrument in the laboratory, with flight baffle plates installed surrounding the aperture and diffuser deployment mechanism. These plates are designed to shield the aperture from both direct solar incident radiation and spacecraft surface reflections of both solar light and earth shine. Most parts facing the aperture are anodized in black, which typically has a low reflectivity of several percent in the UV wavelength range of interest (Lowery, 1977). The noticeably brighter horizontal line in the picture is the cylindrical axle of the diffuser deployment mechanism, which reflects roof light into the camera. When the axle is illuminated with a flood light at different angles, the camera can capture the light on a different part of the axle due to the cylindrical geometry. The axle is a couple of 


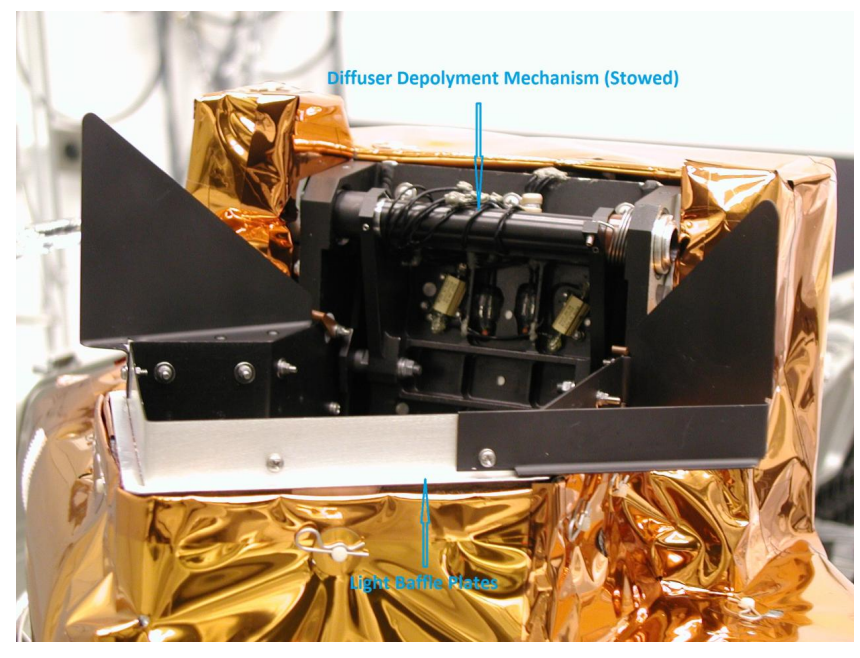

Fig. 4. SBUV/2 instrument in the laboratory. Pieces of anodized plates are installed surrounding the SBUV/2 aperture as light baffles. The diffuser deployment mechanism has a cylindrical axle that reflects roof light into the viewer's eye. Courtesy of Ball Aerospace and Technologies Corp.

centimeters above the front baffle in the picture. In orbit, the aperture is oriented downward towards Earth, and the axle is below the front baffle. When the satellite moves from the daylight to the nightside near terminator, the diffuser deployment mechanism faces the sun. At $\mathrm{SZA}=75^{\circ}$, solar light can graze over the edges of the baffle plates, and illuminate a portion of the axle toward the depolarizer. The entrant slit of the monochromator, $1.3 \mathrm{~mm} \times 30 \mathrm{~mm}$ in size, is behind the depolarizer. Light scattering and reflection from optical surfaces of the depolarizer can redirect some of the light into the entrance slit within the field of view. This geometry is consistent with the finding of IBSL error onset and rapid rising around $\mathrm{SZA}=77^{\circ}$ near the sunset, shown in Fig. 2. It also explains the clean radiance signal at sunrise when the diffuser deployment mechanism turns its back against the sun. SBUV/2 instruments use a depolarizer, which consists of 4 quartz wedges. In the original instrument design, the surfaces of the 4 quartz wedges are optically contacted with each other. During thermal vacuum testing of N16 in 1987, two of the quartz crystal surfaces separated causing a sharp change in the instrument's sensitivity, which was later fixed. To avoid further similar problems, the last 4 SBUV/2 instruments manufactured, which flew on the N14, N17, N18 and N19 spacecraft, were modified to have $1 \mathrm{~mm}$ air gaps between the surfaces of the depolarization wedges. Apparently, the air-gapped quartz wedges have surface scattering and reflection 1 order of magnitude larger than the optically contacted surfaces, which results in significant IBSL errors. Note that the CCR does not have the IBSL problem because its aperture is not covered by the depolarizer. The IBSL problem was first uncovered in N17 orbital data analysis as early as 2004. However, the last two SBUV/2 instruments to be
Initial Profile Albedo Residue Difference: N19 - N17 (2009163)
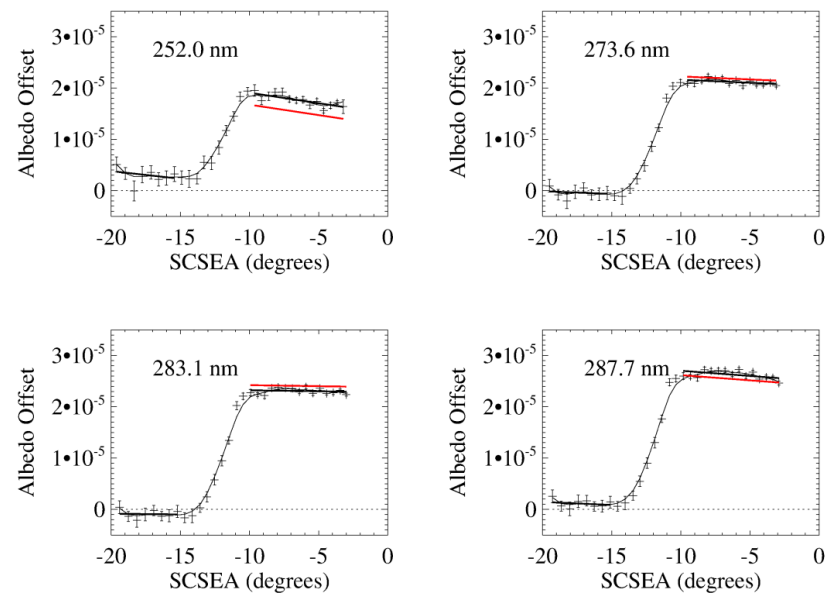

Fig. 5. Initial profile albedo residue (IPAR) comparison between NOAA-19 and NOAA-17 in the Northern Hemisphere, where N19 is contaminated with IBSL and N17 is clean. The comparison is made at the same latitude band of $0.5^{\circ}$ on a weekly average. The difference is plotted against N19's spacecraft centered solar elevation angle (SCSEA). Under normal satellite attitude conditions, the SCSEA is equal to $\mathrm{SZA}-90^{\circ}$. Therefore, these figures cover the dayside from $\mathrm{SZA}=70^{\circ}$ to $\mathrm{SZA}=90^{\circ}$. Standard errors in the average are plotted as error bars. IPAR differences, weighted with $1 /$ error $^{2}$, are fitted separately with two straight lines at SCSEA $\geq-10^{\circ}$ and SCESA $\leq-15^{\circ}$, shown with solid, thick black lines. The fitted IPAR is subtracted with the background at $-15^{\circ}$, shown in red.

launched on board N18 and N19 could not be modified because of both flight schedules and budgetary constraints. In addition, there was no opportunity to perform laboratory tests to fully characterize the IBSL error before flight. Therefore, we have to use on-orbit SBUV/2 measurements to characterize the IBSL error.

\section{Characterization of IBSL}

\subsection{Dayside}

We have identified the IBSL error in Fig. 2 using direct comparison of N16 and N18 albedo measurements at $273 \mathrm{~nm}$. These instruments were in similar orbits in 2005, which reduces possible differences in measured radiances due to diurnal ozone variations. Otherwise, the measured radiances as a function of SZA by two instruments at different orbits can be very different in their field of views, scene reflectivity, terrain height and other atmospheric and geophysical properties. In the V8 SBUV ozone retrieval, an a priori ozone profile is constructed as an initial estimate of the ozone profile, which has the total column ozone equal to the total column ozone determined with measurements at $318 \mathrm{~nm}$ for ozone absorption and at $331 \mathrm{~nm}$ for scene reflectivity (Bhartia et al., 2013). The initial ozone profile is 
used to calculate albedo values at other short wavelengths in the same spectral scan, using the atmospheric backscattering forward model. The difference between the measurement and the computed albedo value, called the initial profile albedo residue (IPAR) in this paper, contains the difference between the actual ozone profile and the initial estimate, the temperature dependent ozone absorption cross-section error, the calibration offset, as well as any IBSL error. Weekly $0.5^{\circ}$ latitude band zonal means of IPAR are computed and compared between two SBUV/2 instruments at the same latitude to minimize both geophysical and atmospheric differences. Figure 5 shows the weekly average differences in IPAR between N19 and N17 near the North Pole as a function of the SCSEA of N19, where N19 approaching sunset was contaminated with IBSL error and N17 at sunrise was not contaminated by IBSL error. Since this error is observed near spacecraft sunset, N17 measurements have significant IBSL errors near the South Pole, while N19 measurements in that region are not contaminated. Therefore, a similar comparison to Fig. 5 near the South Pole would reveal the N17 IBSL error. The spacecraft centered coordinates, SCSEA and spacecraft centered solar azimuth angle (SCSAA), are preferred for the IBSL analysis over the reference frame on the Earth's surface, since the IBSL error is attributed to the direct solar incidence on the spacecraft rather than earth shine. We will demonstrate the strong correlation between IBSL and SCSAA in the next sections, whereas there is no direct correlation between solar azimuth angle (SAA measured at the surface clockwise from due north) and IBSL. The IBSL error rises rapidly in the region between SCSEA $=-15^{\circ}$ and SCSEA $=-10^{\circ}$ (corresponding to $\mathrm{SZA}=75^{\circ}$ and $80^{\circ}$ ), and flattens between SCSEA $=-10^{\circ}$ and SCSEA $=-2^{\circ}$ (corresponding to $\mathrm{SZA}=80^{\circ}$ and $88^{\circ}$ ). The error bars are the standard errors for the latitude band zonal means. Note also the offset of $3 \times 10^{-6}$ at $273 \mathrm{~nm}$ at SCSEA $=-15^{\circ}$, which is about $3 \%$ of measured albedo value. This albedo offset can be a combination of calibration offsets in both N19 and N17, which have a $1 \%$ uncertainty (DeLand et al., 2012), as well as possible diurnal changes in ozone profiles due to the different local times of the measurements (Haefele et al., 2008). The offset fluctuated from week to week at different wavelengths from $-2 \times 10^{-6}$ to $7 \times 10^{-6}$. This is larger than the likely calibration offset or instrument noise effect, and thus is likely due to atmospheric and geophysical differences.

The IPAR differences, weighted with the reciprocal of the squared standard error values, are fitted with two straight lines separately over two SCSEA ranges, $\left[20^{\circ},-15^{\circ}\right]$ and $\left[-10^{\circ},-8^{\circ}\right]$, as shown by the thick, solid black lines. The fitting gives IPAR differences at SCSEA $=-10^{\circ}$ and SCSEA $=-15^{\circ}$. We estimate the IBSL error at SCSEA $=-10^{\circ}$ by subtracting the IPAR difference at SCSEA $=-15^{\circ}$ from the difference at SCSEA $=-10^{\circ}$, which is plotted in red. We also tried to use extrapolation of a straight line fitted between SCSEA $=-20^{\circ}$ and SCSEA $=-15^{\circ}$ for the offset subtraction, but found that the weekly average results were too noisy

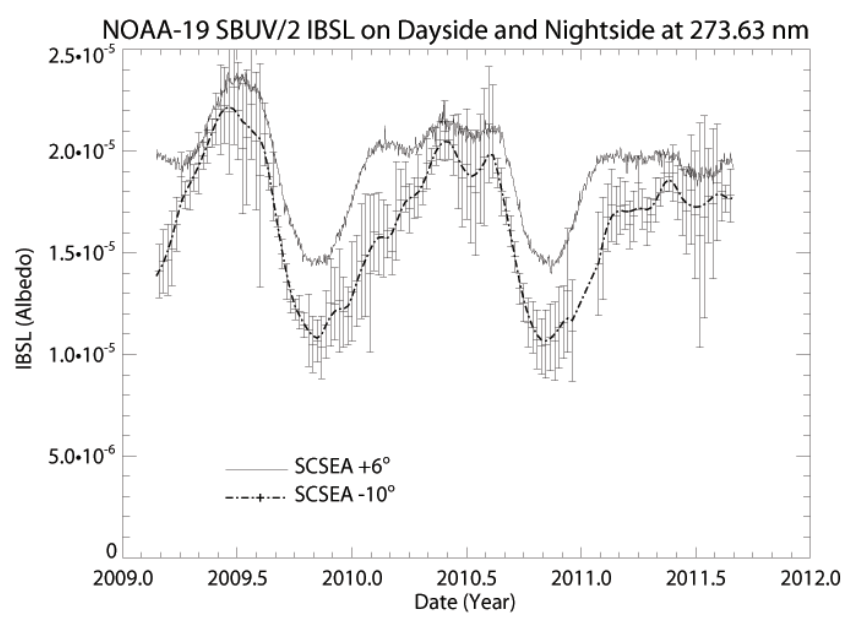

Fig. 6. N19 IBSL error values at $273 \mathrm{~nm}$ at SCSEA $=-10^{\circ}$ and SCSEA $=6^{\circ}$. At SCSEA $=-10^{\circ}$, the dayside weekly averages are derived from comparison of IPAR with N17. The noise (standard errors) in the weekly $0.5^{\circ}$ latitude zonal means of the IPAR differences propagated through the linear regression resulted in uncertainty in the estimated IBSL errors. Then, a piecewise linear fit is used to smooth and interpolate the weekly values. At SCSEA $=6^{\circ}$, N19 IBSL errors are derived directly from daily earth view measurements on the nightside (see Sect. 3.2), which have standard errors of around $0.3 \%$.

to be acceptable. We do not use this IPAR difference to determine the IBSL error at SCSEA $>-10^{\circ}$ beyond the initial step-up point because of the large uncertainty in the offset subtraction. We limit the use of the dayside data to channels 2-4 (273.5, 283.1, 287.6 nm). Channel 1 is not included in this analysis because measurement noise is much more significant due to the low signal level at $252 \mathrm{~nm}$. Determination of IBSL error at the longer wavelengths (channels 5 and up) is more difficult because the measurements are impacted more by surface reflectivity variations and other differences in atmospheric properties in the lower atmosphere. The IPAR comparison can also be limited seasonally because of variations in orbital geometry. The observed N19 IBSL error values at SCSEA $=-10^{\circ}$ are plotted as a function of time in Fig. 6. The standard errors in the weekly $0.5^{\circ}$ latitude band zonal means are propagated through the linear regressions, and produce the uncertainties for the IPAR differences. The estimated uncertainty in the dayside weekly IBSL errors varied from 5 to $10 \%$, as plotted in error bars.

Figure 7 shows 2 yr of data points in the IPAR zonal mean comparison at $273 \mathrm{~nm}$ between N19 and N17. The thin solid curve is derived from a piece-wise linear fit $\left(\triangle \operatorname{SCSEA}=1^{\circ}\right)$ weighted with the reciprocal of the square of the standard error. Note that this curve is not necessarily centered with respect to the spread of data points because of the weighting. A straight line is then fit to the IPAR difference between SC$\mathrm{SEA}=-20^{\circ}$ and SCSEA $=-15^{\circ}$, and extrapolated to correct the offset. The corrected fit result is plotted as a thick 


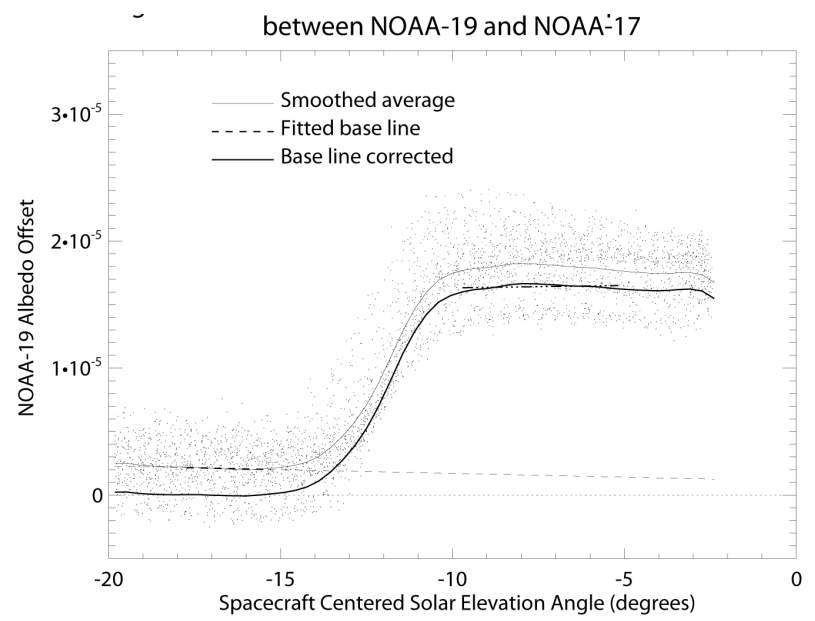

Fig. 7. Two years of data points from IPAR comparisons between N19 and N17 in the Northern Hemisphere illustrate the IBSL error at $273 \mathrm{~nm}$ as a function of SCSEA, a rapid rising edge and flat top before and after SCSEA $=-10^{\circ}$. Piece-wised linear fitting, weighted with statistical error of each point, is used to get a smooth average (thin solid curve). Then, it is corrected for background offset which is an extrapolation of a straight line fitted between SC$\mathrm{SEA}=-20^{\circ}$ and SCSEA $=-15^{\circ}$ (dashed line). The final result for IBSL is plotted as a thick, solid curve.

solid curve. Two results come from this exercise. First, the IBSL error between SCSEA $=-10^{\circ}$ and SCSEA $=-2^{\circ}$ can be approximated well with a straight line. We will extend this linear approximation to SCSEA $=6^{\circ}$ for further analysis. Second, the smooth curve between SCSEA $=-15^{\circ}$ and SCSEA $=-10^{\circ}$ provides the shape of the rising edge of the IBSL error.

\subsection{Nightside}

As discussed above, the use of dayside data is limited to IBSL errors at SCSEA $=-10^{\circ}\left(\mathrm{SZA}=80^{\circ}\right)$ at 3 short wavelengths from 273 to $289 \mathrm{~nm}$, and determination of the IBSL shape (rapid rising edge and relatively flat top) as a function of SCSEA. To model the behavior of the dayside IBSL error for all conditions, we have to look further into the nightside, where both the SCSAA and SCSEA dependence of the IBSL error can be accurately measured and characterized. Figure 8 shows typical nightside IBSL data measured by N19 in just one day. The nightside measurements are calibrated in the same way as the albedo measurements in daylight (DeLand et al., 2012). At short wavelengths from 252 to $298 \mathrm{~nm}$, the earth shine signal drops rapidly to negligible level at SCSEA $=6^{\circ}\left(\mathrm{SZA}=96^{\circ}\right)$ as measured near the South Pole where N19 does not have an IBSL error. At wavelengths longer than $301 \mathrm{~nm}$, earth shine decreases slowly to a negligible level at SCSEA $=9^{\circ}\left(\mathrm{SZA}=99^{\circ}\right)$. The magnitude of the nightside IBSL error from 252 to $340 \mathrm{~nm}$ increases by a factor of 2 in terms of albedo, compared to
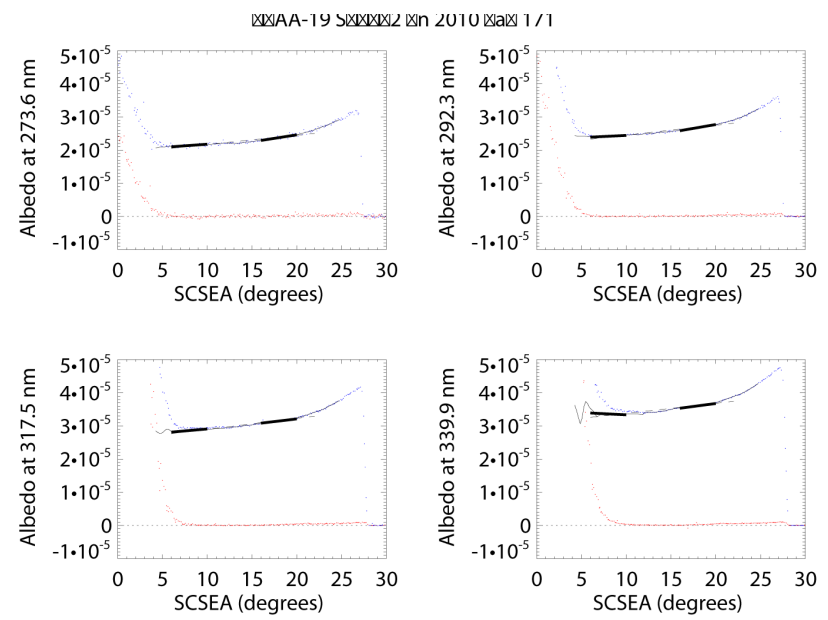

Fig. 8. Example of N19 IBSL nightside daily measurements on the nightside on day 171 of 2010. Larger IBSL errors were measured in the Northern Hemisphere in blue, and lower signals are for the Southern Hemisphere, in red. Thin solid curves are for IBSL after the empirical correction of earth shine near terminator. Thick straight lines are respectively fitted between SCSEA $=6^{\circ}$ and $10^{\circ}$ for daily IBSL at SCSEA $=6^{\circ}$, and between SCSEA $=16^{\circ}$ and $20^{\circ}$ for daily IBSL at SCSEA $=18^{\circ}$.

the larger spectral dependence of the dayside albedo. This weaker wavelength dependence confirms the assumption that the IBSL error is caused by some metal surface reflection of direct solar irradiance rather than earth shine. IBSL contamination in the albedo measurements at wavelengths longer than $318 \mathrm{~nm}$ is negligibly small $(<0.1 \%)$ because of the 2 orders of magnitude increase in Earth albedo at these wavelengths (Fig. 1). Similarly, there is a negligible IBSL error in the solar irradiance measurements, because the minimum solar irradiance signal at all wavelengths is at least 4 orders of magnitude larger than the IBSL error.

The IBSL error is not necessarily a linear function of SCSEA. As shown in Fig. 8, IBSL can be fitted well (within $3 \%$ of the overall error) between SCSEA $=6^{\circ}$ and SCSEA $=22^{\circ}$ with a straight line. However, it can deviate significantly from extrapolations of the fitted line at SCSEA $=27^{\circ}$, particularly when SCSAA is high $\left(70^{\circ}\right.$ in the example). To reduce uncertainty, we choose IBSL measurements at SCSEA $=6^{\circ}$, as close as possible to the dayside, for interpolation of the IBSL error at short wavelengths. However, the full use of IBSL measurements at SCSEA $=6^{\circ}$ will be limited due to earth shine at the long wavelengths. We will first study the IBSL error at SCSEA $=18^{\circ}$, where the measurements have sufficient accuracy, to understand its time dependence, goniometric dependence and wavelength dependence. 

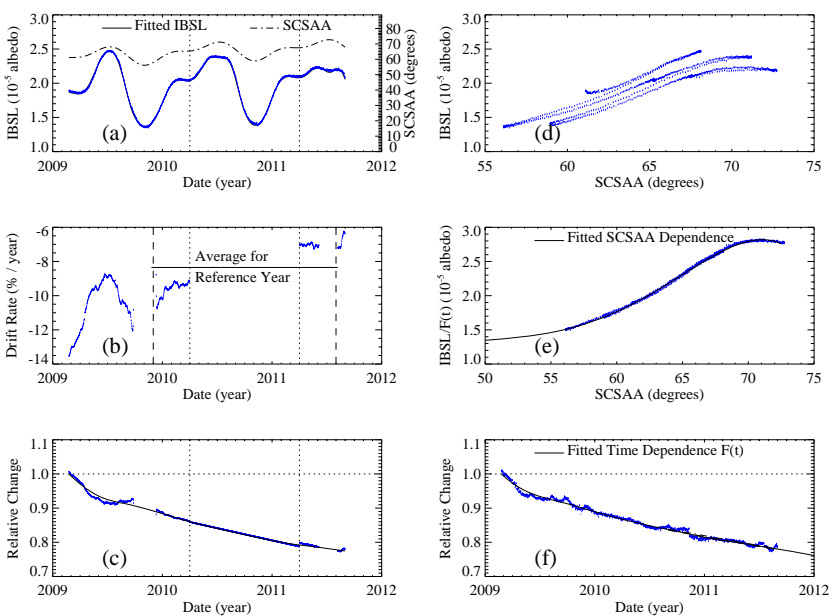

Fig. 9. N19 IBSL at $273 \mathrm{~nm}$ at $\mathrm{SCSEA}=18^{\circ}$ is a product of time dependence and SCSAA dependence. (a) Daily IBSL values (blue dots for raw data) are highly correlated with SCSAA (dashed curve), and can be represented by $F(t) \times G$ (SCSAA) (solid curve). (b) Average drift rates are derived from comparison of daily IBSL values at the same SCSAA. Missing data are due to SCSAA values outside the range in the reference period. (c) Initial estimate of IBSL time dependence is derived from the comparison of daily IBSL values at the same SCSAA, and from the average drift rate for the reference period. It is smoothed and interpolated with piecewise linear fit. (d) Daily IBSL values before removing the time dependence are plotted against SCSAA at SCSEA $=18^{\circ}$. (e) $\operatorname{IBSL} / F(t)$ is fitted with a polynomial of SCSAA, $G$ (SCSAA). (f) Relative changes are reiterated with daily IBSL values divided by the fitted $G$ (SCSAA), then normalized and smoothed for the time dependence function, $F(t)$.

\subsubsection{Separation of time dependence and goniometric dependence}

A linear fit of daily IBSL measurements between SC$\mathrm{SEA}=14^{\circ}$ and $\mathrm{SCSEA}=22^{\circ}$ gives values for both the IBSL error and the rate of change dIBSL/dSCSEA at SC$\mathrm{SEA}=18^{\circ}$. Figure $9 \mathrm{a}$ shows the derived daily IBSL error at SCSEA $=18^{\circ}$ at $273 \mathrm{~nm}$ as a function of time (dots) for N19. The seasonal variations have a strong correlation with variations in SCSAA (dashed curve). Since the reflectivity of surfaces near the instrument entrance slit may change with time, and the illumination geometry of the SBUV/2 instrument will vary with season, the overall IBSL error is assumed to be a product of a time dependence function and a goniometric function:

$\operatorname{IBSL}(t, \varphi)=F(t) \times G(\varphi)$,

where $t$ is time, $\varphi$ is SCSAA, and the wavelength dependence in $F$ and $G$ is implied. Note that $\varphi$ is also a function of time, and $\varphi, F$ and $G$ are all considered at SCSEA $=18^{\circ}$. In order to derive the time-dependent factor $F(t)$, we first compare each daily IBSL measurement with one at the same SCSAA in a reference year, defined as April 2010 to March 2011:

$R_{i}(t)=\operatorname{IBSL}(t, \varphi) / \operatorname{IBSL}\left(t_{\mathrm{r}}, \varphi\right)=F(t) / F\left(t_{\mathrm{r}}\right)$,

where $t_{\mathrm{r}}$ is the time in the reference year, and Eq. (1) is used to eliminate the goniometric factor. For a given set of $t$ and $\varphi$, two consecutive days in the reference year are selected for having SCSAA adjacent to $\varphi$, and their IBSL errors are interpolated as a function of $\varphi$ to obtain $\operatorname{IBSL}\left(t_{\mathrm{r}}, \varphi\right)$, where $t_{\mathrm{r}}$ is an interpolated time between these two consecutive days. Depending on the season, as many as three periods in the reference year may occur with adjacent $\varphi$ values. We select the period with the largest time interval. Then, normalized drift rates are calculated,

$\Delta R_{i}(t) / \Delta t=\left(R_{i}(t)-1\right) /\left(t-t_{\mathrm{r}}\right)$.

The results are plotted in Fig. 9b. No drift rates in the reference year are included to avoid large noise associated with the small time interval. There are missing days in the figure because some SCSAA values are outside the range of the reference period. The average drift rate over 4 months before April 2010 and 4 months after March 2011 is calculated to determine the drift rate during the reference period, shown by the solid line. This allows us to make an initial estimate of the IBSL time dependence during the reference period, using a linear approximation. An initial estimate of IBSL time dependence can then be obtained with

$f(t)=\left[1+\left(t-t_{\mathrm{b}}\right) \times\left\langle\Delta R_{i}(t) / \Delta t\right\rangle\right] \times R_{i}(t) \approx F(t) / F\left(t_{\mathrm{b}}\right)$,

where $\left\langle\Delta R_{i}(t) / \Delta t\right\rangle$ is the average drift rate during the reference year, $t_{\mathrm{b}}$ is chosen to be at the beginning of the reference year for convenience, and the " $\approx$ " sign could be replaced with the "=" sign if the drift in the reference year is strictly linear. Thus far, the time dependence function for IBSL is normalized at $t_{\mathrm{b}}$. We renormalize it to the first day of N19's earth radiance measurements,

$F_{n}(t) \equiv f(t) / f\left(t_{1}\right) \approx F(t) / F\left(t_{1}\right)$,

where $t_{1}$ is the time of the first day of N19's radiance measurements. The resulting $F_{n}(t)$ values are shown by the dots in Fig. 9c. Note that $F_{n}(t) \approx F(t)$ will be valid when we choose $F(t)$ in Eq. (1) to be normalized on day one. These $F_{n}(t)$ values are smoothed and interpolated with a piece-wise linear fit to get an initial estimate of the time dependence, shown as the solid curve. The IBSL daily values shown in Fig. 9a can also be plotted as a function of SCSAA, as shown in Fig. 9d. Dividing these daily IBSL values by the $F(t)$ function derived in Fig. 9c gives us the IBSL SCSAA dependence, which is now a well behaved function, as shown by the dots in Fig. 9e. A 4th order polynomial function fits these data well, as shown by the smooth curve, which will be used as $G(\varphi)$ in Eq. (1). The fitted $G(\varphi)$ function is then used to remove the SCSAA dependence from the daily IBSL measurements. This creates a new estimate of the IBSL time 


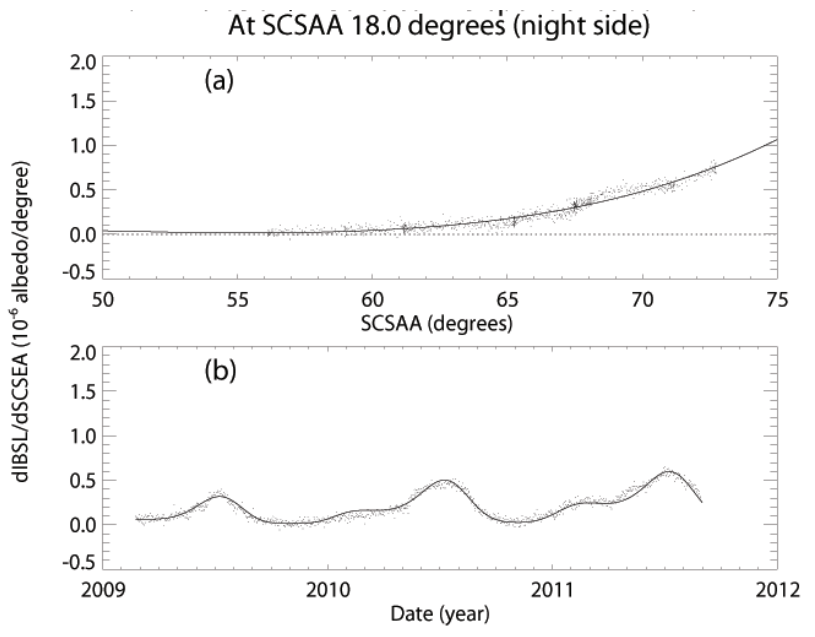

Fig. 10. (a) dIBSL / dSCSEA/F(t) can be fitted with a polynomial of SCSAA, $S$ (SCSAA). (b) Daily values of the slope dIBSL / dSCSEA as a function of time (dots) can be approximated with $F(t) \times$ $S(\operatorname{SCSAA}(t))$ (solid curve).

dependence using all measurements, as shown by the dots in Fig. 9f. These values are smoothed to determine the time dependence function $F(t)$. This process is iterated once. The final IBSL time dependence is shown with the smooth curve in Fig. 9f. Combining the IBSL time dependence and the IBSL SCSAA dependence from Eq (1), we can calculate the IBSL error at SCSEA $=18^{\circ}$, shown by the smooth curve in Fig. 9a, where SCSAA is measured at SCSEA $=18^{\circ}$ and is approximately constant in a given day.

Daily values of dIBSL/dSCSEA at $273 \mathrm{~nm}$ are plotted as a function of time in dots in Fig. 10b. We find that dIBSL/dSCSEA is also correlated with SCSAA. The normalized slope is shown as a function of SCSAA in Fig. 10a, where we have removed the time dependence $F(t)$ shown in Fig. 9f. The normalized slope can be then fitted with a polynomial function, $S(\varphi)$, shown by the smooth curve in Fig. 10a. Thus, we can reproduce the time dependence of the slope with the fitted functions,

$\operatorname{dIBSL} / \operatorname{dSCSEA}(t)=S(\varphi(t)) \times F(t)$,

which is shown by the smooth curve in Fig. 10b.

\subsubsection{Goniometric functions at $\operatorname{SCSEA}=18^{\circ}$}

All 12 wavelength channels of daily IBSL values at SC$\mathrm{SEA}=18^{\circ}$ are processed to derive their goniometric functions in the same way as described in the previous section. Figure 11a shows all 12 fitted $G(\varphi, \lambda)$ functions at SC$\mathrm{SEA}=18^{\circ}$ for N19. Since the time dependence functions, $F(t, \lambda)$, are normalized on the first day of N19's earth view radiance measurements, $G(\varphi, \lambda)$ gives IBSL at SC$\mathrm{SEA}=18^{\circ}$ on that date when $\varphi=61.1^{\circ}$. The fitted polynomials in Fig. 11a are very similar to each other in shape. They
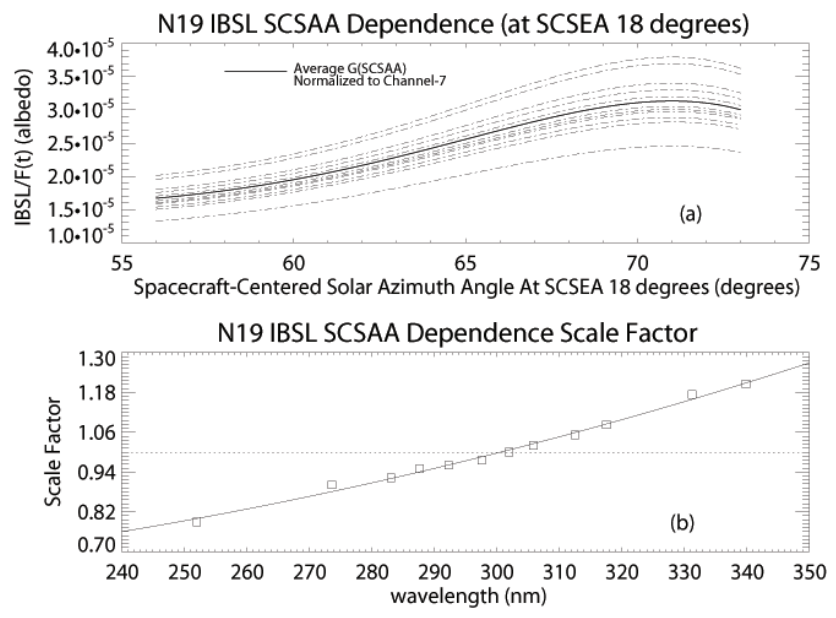

Fig. 11. Dashed curves in the top panel are for 12 channels of fitted $G$ (SCSAA) functions, which can be approximated within $0.7 \%$ of IBSL by a product of a wavelength independent factor for the SCSAA dependence and the scale factors in the bottom panel, $g_{0}(\mathrm{SCSAA}) \times C(\lambda)$.

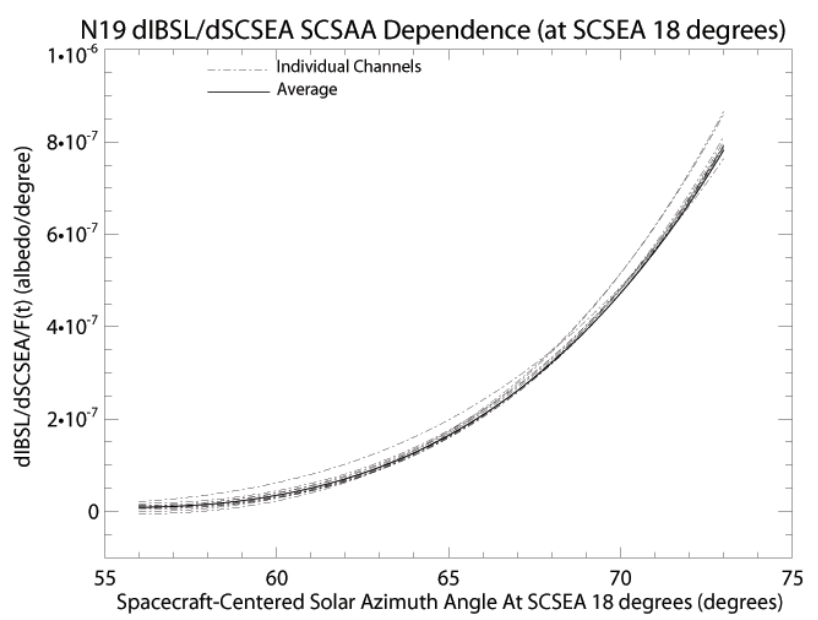

Fig. 12. Fitted $S$ (SCSAA) functions for the IBSL slope, dIBSL / dSCSEA, at different wavelengths can be approximated with an average (solid line).

can be approximated by

$g(\varphi, \lambda)=g_{0}(\varphi) \times C(\lambda)$,

where $g_{0}(\varphi)$ is an average of $G(\varphi, \lambda)$ over all wavelengths, shown as the thick solid curve, and SCSAA is taken at SC$\mathrm{SEA}=18^{\circ}$. The scale factors $C(\lambda)$, shown in Fig. $11 \mathrm{~b}$, then minimize the standard deviation between the scaled observations and the average. The differences between $G(\varphi, \lambda)$ and $g(\varphi, \lambda)$ are less than $0.7 \%$ of the IBSL error, except for channel 1 with a maximum of $1.2 \%$ due to low signal level at $252 \mathrm{~nm}$. Therefore, $g(\varphi, \lambda)$ is a valid approximation of the IBSL error's goniometric dependence at SCSEA $=18^{\circ}$. 
Figure 12 shows the fitted $S(\varphi)$ functions for the slope dIBSL/dSCSEA normalized by the time dependence $F(t)$ at all 12 wavelengths. We can see that $S(\varphi)$ is essentially wavelength-independent. An average of $S(\varphi)$ is computed (thick solid curve). A maximum difference of $10^{-7}$ at SC$\mathrm{SAA}=73^{\circ}$ is found between the average and channel 12 , which is less than $0.5 \%$ of the IBSL error. Therefore, an average of the fitted $S(\varphi)$ functions can be used for the goniometric dependence of the slope at all wavelengths.

From the above exercises we conclude that the goniometric functions for the IBSL and dIBSL/dSCSEA can be approximated as the combination of a wavelength-independent angular function and a wavelength-dependent scale factor. This is because the light baffle shape determines how the baffle blocks the solar light in various solar incidence angles, which is purely geometrical. The reflection of illuminated surfaces can also have both the wavelength dependence and the angular dependence. The separation of the wavelength dependence and the angular dependence, as described above, provides a convenient and suitable approximation for the practical purpose of IBSL correction.

\subsubsection{IBSL at SCSEA $=6^{\circ}$}

The radiance measurements before the sunrise in the Southern Hemisphere, by N19 near the South Pole as the example in Fig. 8, show how ozone absorption blocks the solar light in the UV spectrum. At $\mathrm{SZA}=99^{\circ}$, the albedo values at $340 \mathrm{~nm}$ in Southern Hemisphere is about $1 \%$ of IBSL found in the Northern Hemisphere, where the earth shine is from light scattering of atmosphere above $90 \mathrm{~km}$. It dropped below the sensor's noise level at SZA $=100^{\circ}$ in Southern Hemisphere, which corresponds to a height of $100 \mathrm{~km}$. (N19 picks up some negligible IBSL in Southern Hemisphere starting at SCSEA $=18^{\circ}$.) Ozone absorption cross sections at wavelengths shorter than $300 \mathrm{~nm}$ are 2-3 orders of magnitude larger than that at $340 \mathrm{~nm}$. Therefore, solar light at the short wavelengths are completely blocked by ozone, rather than by an Earth eclipse, at much lower altitude before reaching $\mathrm{SZA}=96^{\circ}$. To estimate IBSL around SCSEA $=6^{\circ}$, an empirical correction of the earth shine after the sunset is attempted, which scales the earth shine measurements before the sunrise with $\mathrm{CCR}$ values at $\mathrm{SZA}=96^{\circ}$ :

$$
\begin{aligned}
& I_{\text {sunset }}(\lambda, \mathrm{SZA})=I_{\text {sunrise }}(\lambda, \mathrm{SZA}) \\
& \times\left[I_{\text {sunset }}\left(\lambda=378 \mathrm{~nm}, \mathrm{SZA}=96^{\circ}\right) /\right. \\
& \left.I_{\text {sunrise }}\left(\lambda=378 \mathrm{~nm}, \mathrm{SZA}=96^{\circ}\right)\right],
\end{aligned}
$$

where $I_{\text {sunset }}$ and $I_{\text {sunrise }}$ are in the albedo values. This is considered as a zero-order approximation. The resulting IBSL after the earth shine correction are plotted in thick solid curves in Fig. 8. This earth shine correction has no impact at the short wavelengths at SCSEA $=6^{\circ}$. At the long wavelengths, residues of earth shine correction contribute to noise in daily IBSL values at SCSEA $=6^{\circ}$, about $1-2 \%$ in terms

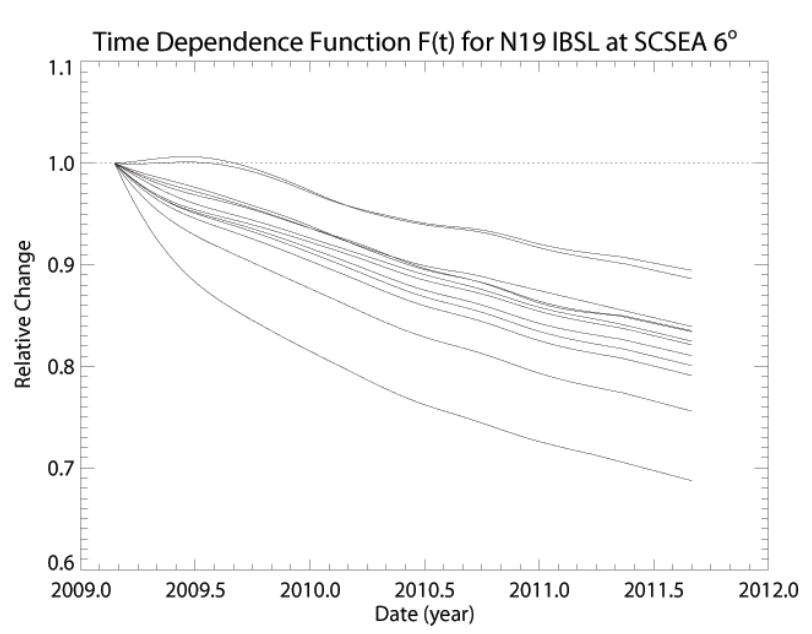

Fig. 13. Time dependence functions $F(t, \lambda)$ for N19 IBSL at SC$\mathrm{SEA}=6^{\circ}$, normalized to the start of the mission. The magnitude of the overall drift monotonically increases towards shorter wavelengths. At the end of the data record, the magnitude of $F(t, \lambda)$ ranges monotonically from 0.68 at $252 \mathrm{~nm}$ to 0.90 at $340 \mathrm{~nm}$.

of standard deviation (with a peak-to-peak noise of $10 \%$ of IBSL), which is negligible in comparison with albedo values in daylight. The daily IBSL values at SCSEA $=6^{\circ}$ are derived from a smooth function fit over SCSEA $=6^{\circ}$ and SC$\mathrm{SEA}=12^{\circ}$. The resulting daily IBSL values at $273 \mathrm{~nm}$ are plotted in Fig. 6.

Estimated daily IBSL values at SCSEA $=6^{\circ}$ are then processed for the separation of time dependence and goniometric dependence terms in the same way as described in Sect. 3.2.1. Figure 13 shows the time dependence functions, $F(t, \lambda)$, at all 12 wavelengths. The results at SCSEA $=6^{\circ}$ are consistent with those at SCSEA $=18^{\circ}$. For example, at $273 \mathrm{~nm}$ the calculated relative IBSL error at SCSEA $=6^{\circ} \mathrm{de}-$ creased to 0.751 at the end of August 2011, compared to a calculated value of 0.775 at SCSEA $=18^{\circ}$ shown in Fig. 9f. Note that long wavelength channels have a lower drift rate than the short wavelength channels. This is a typical behavior in metal surface reflectivity in the UV spectrum in the processes of photo carbonization of prelaunch contamination on the spacecraft (Hilsenrath et al., 1994).

The calculated SCSAA dependence of the IBSL error as a function of wavelength at SCSEA $=6^{\circ}, G(\varphi, \lambda)$, is plotted in Fig. 14a. These curves share a similarity in shape consistent with Fig. 11a. However, only the short wavelength channels 2-6 are close to each other in shape to a high degree of accuracy $(0.7 \%)$. Large differences are found with the other channels due to the limitation at SCSEA $=6^{\circ}$, earth shine contamination at long wavelengths and low solar flux at $252 \mathrm{~nm}$. However, the percentage of IBSL errors at the long wavelengths is negligibly small, and the $252 \mathrm{~nm}$ channel is not used in the V8.6 SBUV/2 profile retrieval (Bhartia et al., 2013). Therefore, we take the average only over channels $2-6$ 

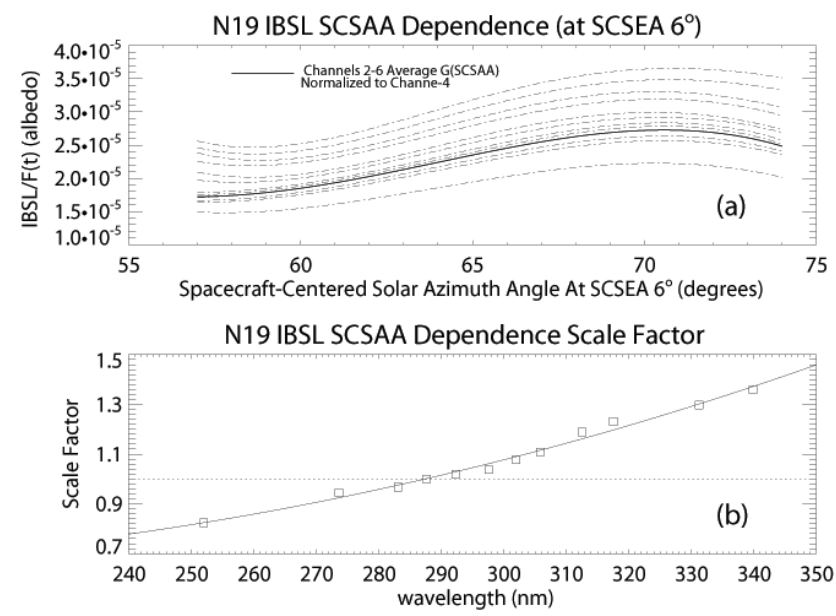

Fig. 14. (a) Dashed curves in the top panel are for 12 channels of fitted $G$ (SCSAA) functions, which monotonically increases as wavelength increases. Only channels 2-6 are averaged for the wavelength independent factor $g_{0}$ (SCSAA) because of the earth shine contamination at the long wavelengths and low signal at $252 \mathrm{~nm}$. (b) The scale factors in the bottom panel, $C(\lambda)$, result from the least square between $G(\mathrm{SCSAA})$ and $g_{0}(\mathrm{SCSAA}) \times C(\lambda)$.

for the wavelength independent shape factor, $g_{0}(\varphi)$, shown with a thick, solid curve. The scale factors, $C(\lambda)$ shown in Fig. 14b, result in least squares of deviation between $g_{0}(\varphi) \times C(\lambda)$ and $G(\varphi, \lambda)$, which are less than $0.25 \%$ in terms of standard deviations for channels $2-6$. Selection of these 5 short wavelength channels for the average guarantee the accuracy for IBSL error corrections in the ozone profile retrieval. We believe this average is a better representation of the goniometric dependence for the other long wavelength channels and that at $252 \mathrm{~nm}$. The wavelength dependence of $C(\lambda)$ at SCSEA $=6^{\circ}$ is characteristically comparable to that at SCSEA $=18^{\circ}$ shown in Fig. 11.

The estimation of the IBSL error in daylight $\left(\mathrm{SZA}<88^{\circ}\right)$ has to be based on interpolation of the derived values at SCSEA $=-10^{\circ}$ and SCSEA $=6^{\circ}$. The weekly IPAR results at SCSEA $=-10^{\circ}$ are smoothed and interpolated to daily values with the piecewise linear fitting function, as shown in Fig. 6 for N19 at $273 \mathrm{~nm}$. The slope, dIBSL/dSCSEA, is then computed with straight lines connecting the two points. Next, the time-independent normalized slope at SC$\mathrm{SEA}=6^{\circ}, S(\varphi, \lambda)=(\mathrm{dIBSL} / \mathrm{dSCSEA}) / F(t)$, is calculated, as shown in Fig. 15 for N19 as an example. As discussed in Sect. 3.1, we can only obtain valid dayside IBSL at the 6 short wavelengths. Among them, channel 1 has low signal, and channels 5 and 6 have some scene noise. Therefore, relatively more reliable results of $S(\varphi)$ can be obtained only with channels $2-4$. The difference in $S(\varphi)$ among channels 2-4 equates to only $2 \%$ of the IBSL error at the midpoint between SCSEA $=-10^{\circ}$ and SCSEA $=6^{\circ}$. Therefore, the wavelength dependence of $S(\varphi)$ between SCSEA $=-10^{\circ}$ and SCSEA $=6^{\circ}$ is negligible at least among these short wavelength channels. This is consistent with the finding at SCSEA $=18^{\circ}$ that the normalized slopes are approximately wavelength independent. Therefore, the average of $S(\varphi)$ over channels $2-4$ is calculated and used to represent the normalized slope at all wavelengths.

\subsection{IBSL corrections}

Based on the discussions and results in the previous sections, we can model IBSL between SCSEA $=-10^{\circ}$ and SC$\mathrm{SEA}=6^{\circ}$ with the following function:

$\operatorname{IBSL}(\operatorname{SCSEA}, t, \lambda)=$

$\left[g_{0}(\varphi(t)) \times C(\lambda)+S(\varphi(t)) \times(\operatorname{SCSEA}-6)\right] \times F(t, \lambda)$,

where the goniometric functions, $g_{0}, C$ and $S$, and the time dependence function, $F$, are derived and defined at SC$\mathrm{SEA}=6^{\circ}$, and SCSAA $=\varphi(t)$ is measured at SCSEA $=6^{\circ}$ and is approximately constant in a given day. An average of the rising edge of the IBSL error between SCSEA $=-15^{\circ}$ and SCSEA $=-10^{\circ}$ over multiple years, which is derived as described in Sect. 3.1, is scaled to be connected with the straight line of IBSL(SCSEA, $t, \lambda$ ) at SCSEA $=-10^{\circ}$.

We have examined data from all SBUV/2 instruments for IBSL errors. N19 leads (a peak IBSL of $2.4 \times 10^{-5}$ at $273 \mathrm{~nm}$ in terms of albedo), followed by N17 $\left(1.9 \times 10^{-5}\right)$, N14 $\left(1.6 \times 10^{-5}\right)$ and N18 $\left(1.2 \times 10^{-5}\right)$. The instruments that use a depolarizer with optically contacted prism elements (N9, N11, N16) have IBSL errors about 20 times less than the instruments that use a depolarizer with air-gapped prisms (N14, N17, N18, N19). Therefore, the IBSL contamination in N9, $\mathrm{N} 11$ and N16 albedo measurements is less than $1 \%$ even at $\mathrm{SZA}=88^{\circ}$, which is not significant for practical purposes. We have used the model described here to develop separate empirical IBSL correction functions for N14, N17, N18 and N19. These corrections are implemented in the ozone processing code for the current NOAA SBUV/2 operational data processing, as well as for the SBUV/2 V8.6 reprocessing product (DeLand et al., 2012, Bhartia et al., 2013). The estimated uncertainty of the IBSL correction is less than $5 \%$ of the error between $\mathrm{SZA}=80^{\circ}$ and $\mathrm{SZA}=88^{\circ}$, which represents about $1 \%$ in the earth radiance measurements at $273 \mathrm{~nm}$.

Figure 16 shows an example of N19 albedo measurements at $273 \mathrm{~nm}$ before and after IBSL correction. The data were taken on the spring equinox 2010 near the North Pole. While the IBSL error was approximately constant in absolute terms $\left(\sim 2 \times 10^{-5}\right.$ between SZA $=80^{\circ}$ and $\left.88^{\circ}\right)$, the percentage contamination in the albedo measurements increases from $\sim 25 \%$ at $\mathrm{SZA}=80^{\circ}$ to $\sim 40 \%$ at $\mathrm{SZA}=88^{\circ}$. Figure 17 compares retrieved ozone profiles before and after the IBSL correction. The impact of uncorrected IBSL error to the retrieved ozone profile above $1 \mathrm{hPa}$ (Layer 16) is more than $30 \%$. While the retrieved total column ozone amount, which depends on the long wavelength channels, is not affected 


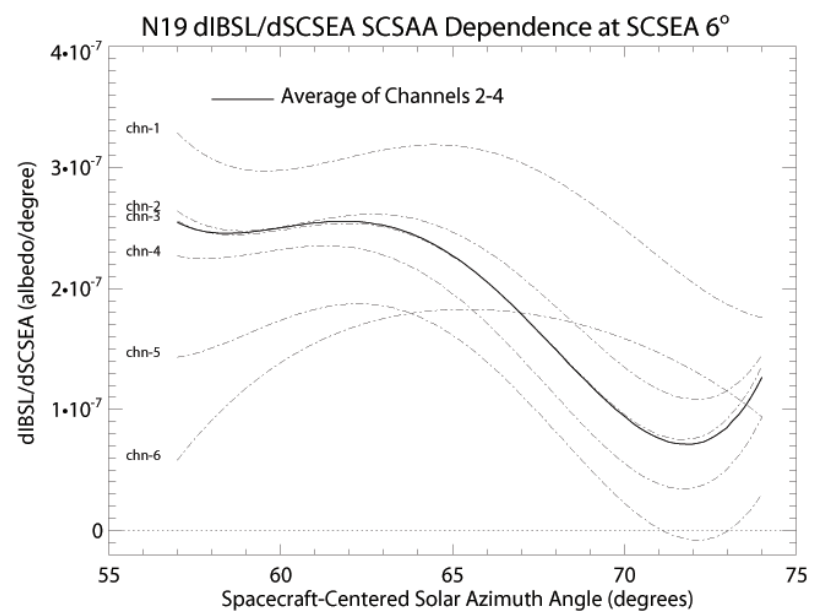

Fig. 15. N19's dIBSL / dSCSEA at SCSEA $=6^{\circ}$ is calculated with IBSL at SCSEA $=-10^{\circ}$ in the IPAR analysis on the dayside and measurements at $\mathrm{SCSEA}=6^{\circ}$ on the nightside.

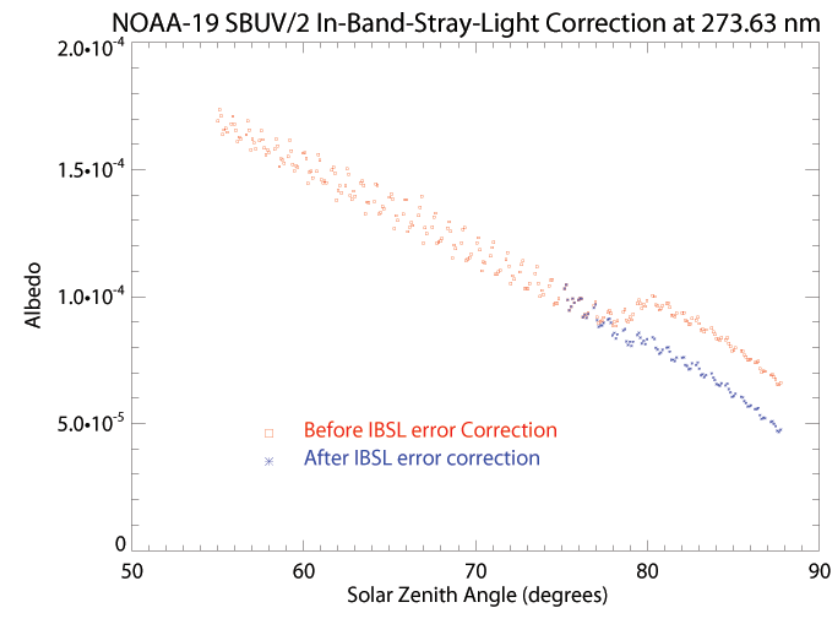

Fig. 16. Sample of N19 albedo measurements at $273 \mathrm{~nm}$ near the North Pole before and after the IBSL correction.

by IBSL, the V8.6 ozone retrieval algorithm pushes up the ozone layers below $10 \mathrm{hPa}$ by $1-2 \%$ to compensate for the deficits in the upper layers. Even though the IBSL error impacts only a small portion of the SBUV/2 data at very high SZA, this error can result in large errors in the retrieved stratospheric ozone values in polar regions. The methodology developed in this paper reduces the IBSL-related uncertainty in the retrieved upper layer ozone to only a few percent.

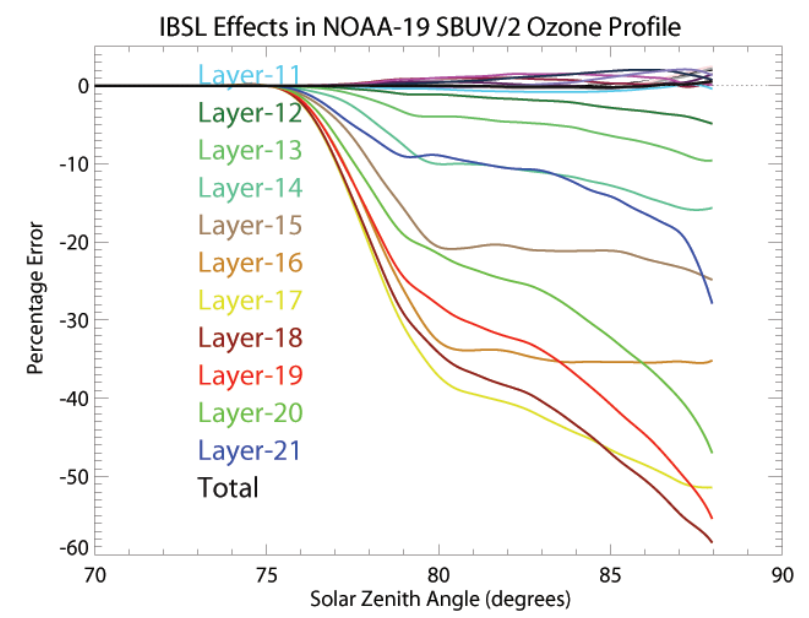

Fig. 17. The sample of IBSL effects in the retrieved ozone profiles near the North Pole is taken from an average of two weeks around the spring equinox 2010. Twenty-one SBUV V8 profile layers are defined by $10^{-2 n}$ atmosphere pressure levels, where $n$ starts from 0 for the bottom boundaries. Only top layers above $10 \mathrm{hPa}$ are labeled. Other lower layers overlap each other within $2 \%$. The black curve for the total column ozone difference is near zero.

\section{Conclusions}

IBSL is found in the earth radiance measurements from the last $4 \mathrm{SBUV} / 2$ instruments, which were flown on the NOAA$14,17,18$ and 19 satellites, at a SZA larger than $77^{\circ}$ when approaching terminator at sunset. The source of IBSL is from direct solar illumination of the spacecraft rather than from earth shine. Large surface reflection and scattering that are associated with the air-gapped depolarizer results in significant IBSL errors. We have characterized the IBSL error between SCSEA $=-10^{\circ}$ and SCSEA $=6^{\circ}$ (corresponding to $\mathrm{SZA}=80^{\circ}$ and $96^{\circ}$ ) with a linear function of SCSEA. The magnitude of IBSL is characterized as a function of both the SCSAA and the mission time. We have implemented an IBSL correction in the V8.6 SBUV/2 ozone retrieval with an uncertainty of $1 \%$ of albedo values. The maximum uncorrected IBSL error can reach $40 \%$ of albedo values at $273 \mathrm{~nm}$ at $\mathrm{SZA}=88^{\circ}$. The retrieved layer ozone above $1 \mathrm{hPa}$ can be underestimated by more than $30 \%$. The IBSL error correction reduces the IBSL-related uncertainty in the retrieved stratosphere ozone profile to a few percent.

Acknowledgements. This work was supported by NASA through contract NNG12HP08C.

Edited by: M. Weber 


\section{References}

Bhartia, P. K., McPeters, R. D., Mateer, C. L., Flynn, L. E., and Wellemeyer, C.: Algorithm for the estimation of vertical ozone profile from the backscattered ultraviolet technique, J. Geophys. Res., 101, 18793-18806, 1996.

Bhartia, P. K., McPeters, R. D., Flynn, L. E., Taylor, S., Kramarova, N. A., Frith, S., Fisher, B., and DeLand, M.: Solar Backscatter UV (SBUV) total ozone and profile algorithm, Atmos. Meas. Tech., 6, 2533-2548, doi:10.5194/amt-6-2533-2013, 2013.

DeFabo, E. C.: Ultraviolet-B radiation and stratospheric ozone loss: potential impacts on human health in the Arctic, Int. J. Circumpolar Health, 59, 4-8, 2000.

DeLand, M. T., Taylor, S. L., Huang, L. K., and Fisher, B. L.: Calibration of the SBUV version 8.6 ozone data product, Atmos. Meas. Tech., 5, 2951-2967, doi:10.5194/amt-5-2951-2012, 2012.

Fiore, A. M., Jacob, D. J., and Field, B. D. Linking ozone pollution and climate change: The case for controlling methane, Geophys. Res. Lett., 29, 25-1, 25-3-25-4, doi:10.1029/2002GL015601, 2002.

Frederick, J. E., Cebula, R. P., and Heath, D. F.: Instrument characterization for the detection of long-term changes in stratospheric ozone: An analysis of the SBUV/2 radiometer, J. Atmos. Oceanic Technol., 3, 472-480, 1986.

Haefele, A., Hocke, K., Kämpfer, N., Keckhut, P., Marchand, M., Bekki, S., Morel, B., Egorova, T., and Rozanov, E.: Diurnal changes in middle atmospheric $\mathrm{H}_{2} \mathrm{O}$ and $\mathrm{O}_{3}$ : Observations in the Alpine region and climate models, J. Geophys. Res., 113, D17303, doi:10.1029/2008JD009892, 2008.

Heath, D. F., Krueger, A. J., Roeder, H. A., and Henderson, B. D.: The Solar Backscatter Ultraviolet and Total Ozone Mapping Spectrometer (SBUV/TOMS) for Nimbus G, Opt. Eng., 14, 323$331,1975$.
Heath, D. F., Wei, Z., Fowler, W. K., and Nelson, V. W.: Comparison of spectral radiance calibrations of SBUV-2 satellite ozone monitoring instruments using integration sphere and flat-plate diffuser techniques, Metrologia, 30, 259-264, doi:10.1088/00261394/30/4/008, 1993.

Hilsenrath, E., Bhartia, P. K., and Cebula, R. P.: Calibration of BUV satellite ozone data - An example for detecting environmental trends, The Earth Observer, NASA, Vol 6, 26-33, 1994.

Hilsenrath, E., Cebula, R. P., DeLand, M. T., Laamann, K., Taylor, S., Wellemeyer, C., and Bhartia, P. K.: Calibration of the NOAA 11 SBUV/2 ozone data set from 1989 to 1993 using in-flight calibration data and SSBUV, J. Geophys. Res., 100, 1351-1366, 1995.

Janz, S., Hilsenrath, E., Butler, J., Heath, D. F., and Cebula, R. P.: Uncertainties in radiance calibrations of backscatter ultraviolet (BUV) instruments, Metrologia, 32, 637-641, 1995.

Lowery, J. R.: Solar absorption characteristics of several coatings and surface finishes, NASA Technical Memorandum, TM X3509, 1977.

McPeters, R. D., Bhartia, P. K., Haffner, D., Labow, G. J., and Flynn, L.: The version 8.6 SBUV ozone data record: An overview, J. Geophys. Res., 118, 8032-8039, doi:10.1002/jgrd.50597, 2013.

Selin, N. E., Wu, S., Nam, K. M., Paltsev, S., Prinn, R. G., and Webster, M. D.: Global health and economic impacts of future ozone pollution, Environ. Res. Lett., 4, 044014, doi:10.1088/17489326/4/4/044014, 2009.

Stolarski, R. S. and Frith, S. M.: Search for evidence of trend slowdown in the long-term TOMS/SBUV total ozone data record: the importance of instrument drift uncertainty, Atmos. Chem. Phys., 6, 4057-4065, doi:10.5194/acp-6-4057-2006, 2006.

Terao, Y. and Logan, J. A.: Consistency of time series and trends of stratospheric ozone as seen by ozonesonde, SAGE II, HALOE, and SBUV(/2), J. Geophys. Res., 112, D06310, doi:10.1029/2006JD007667, 2007. 